\title{
Imagined perspective-changing within and across novel environments
}

\author{
Marios N. Avraamides ${ }^{1}$ and Jonathan W. Kelly ${ }^{2}$ \\ ${ }^{1}$ Department of Psychology, University of Cyprus, P.O. Box 20537, \\ 1678 Nicosia, Cyprus \\ mariosav@ucy.ac.cy \\ ${ }^{2}$ Department of Psychology, University of California, \\ Santa Barbara, CA 93106-9660, USA \\ j_kelly@psych.ucsb.edu
}

\begin{abstract}
Results suggesting that changing perspective and switching across spatial environments held in memory are processes that take place in parallel were obtained from a task-switching experiment. Participants learned layouts of objects in two virtual rooms and then were asked to use their memories to locate the objects from various imagined viewing perspectives. Results revealed that, even after experiencing multiple perspectives, participants maintained viewpoint-dependent memories for the layouts, and that the latencies for changing perspective within and across environments followed a different pattern depending on whether participants imagined adopting the preferred view.
\end{abstract}

\section{Introduction}

Everyday tasks, such as giving and taking route directions, often require imagined navigation within spaces that are not perceptually available. In order to comprehend route directions, for example, we often perform a mental simulation of our movement, imagining ourselves passing through various neighborhoods and intersections that are held in memory.

Past research on spatial memory has suggested that we represent the world in our memory in a hierarchical fashion. That is, we parse the world into smaller meaningful units, creating thus a collection of separate representations (Hirtle \& Jonides, 1985; McNamara, 1986). A hierarchy of representations is then created by linking these representations to higher-order representations (e.g., the separate representations for the rooms of a building are connected to a representation for the building)

Supporting accounts of hierarchical organization of spatial memory, a number of studies (e.g., Stevens \& Coupe, 1978) have shown that our spatial memory is often biased by the use of information stored at a superordinate level in the hierarchy. Furthermore, other studies have provided evidence for the presence of multiple separate spatial representations. McNamara (1986), for example, has demonstrated that spatial judgments about targets in the same spatial region are faster than judgments about 
targets in different spatial regions, suggesting that the knowledge is organized in separate spatial representations.

The hierarchical organization of spatial memory also predicts that mentally switching from one spatial representation to another entails some cognitive effort, since switching across representations requires activating a new spatial representation and possibly inhibiting the previous one.

Indeed, past research has demonstrated this cost for switching between mental representations of different environments. In a study using the cued task-switching paradigm, Brockmole and Wang (2002) had college professors locate, from memory, objects in two nested environments (their office and the building in which their office was located). The subjects were physically located in a separate environment, and their imagined perspective was held constant. Trials probed unpredictably one or the other environment, thus producing a sequence of trials in which the probed environment was the same or different from that of the previous trial while the imagined global perspective was held constant. Results revealed that participants were slower on trials that required mentally switching to a new environment.

However, performing a spatial task such as imaginally following route directions requires not only mentally switching across environments, but also mentally shifting perspective within them. The present study examines the relation between the processes of mentally switching from one imagined environment to another and that of changing imagined perspective.

Prior research on imagined navigation has shown that, even in the absence of landmarks, changing perspective is time demanding (e.g., Avraamides \& Carlson, 2003). This is, of course, expected if mental operations are believed to evoke the perceptualmotor mechanisms that apply to real situations (Jeannerod, 1995; Kosslyn, 1994; Wexler, Kosslyn, \& Berthoz, 1998). Furthermore, when responses such as locating objects from imagined perspectives are mandated, additional cognitive effort -- and hence time -- is needed. In order to be able to localize a target after having moved (physically or mentally) to a new perspective, one needs to update that target's position relative to one's self. A number of studies have established that doing so is harder when the movement is imagined rather than real (e.g., Avraamides, 2003; Klatzky, Loomis, Beall, Chance, \& Golledge, 1998; Presson \& Montello, 1994). Furthermore, Rieser (1989) has shown that while the latency for locating objects after imagined rotations of the viewer increases monotonically with the extent of the rotation, it remains constant with physical rotations.

While both perspective-changing and environment-switching are associated with temporal costs, it is unclear whether those costs are independent of each other. If, for example, one adopts a new imagined perspective in a new environment, is the total temporal cost simply the sum of the two costs? This should be expected if there are two processes that take place sequentially. Alternatively, if switching environment and changing perspective can be carried out in parallel, then the total time needed to do both should depend on the time needed to complete the more difficult of the two. To be more precise, a race model (e.g., Logan, 1988) would predict that the time needed to perform both mental actions should be, on average, somewhat longer than the duration of the longest action, given that the distributions of latencies for the two actions are likely to overlap. Finally, a third possibility is suggested by Brockmole 
and Wang (2003): the cost for changing perspective could be smaller when switching environments than when remaining in the same environment.

Evidence for the latter possibility was provided by Brockmole and Wang (2003). Using a similar procedure to their earlier study, they had participants judge from memory the relative locations of targets from differing imagined perspectives in two familiar environments. In a within-environment block, trials probed targets from only one of the environments but randomly from two different perspectives. This produced a string of trials in which the perspective could be the same or different from the preceding trial. In an across-environment block, each of the two environments was fixed to a specific (but unique) perspective. Trials probed objects from the two environments in a random manner, thus producing a sequence of trials in which the environment (and, as a result, the perspective) could have either remained the same or changed from trial to trial.

Results revealed that the cost for changing perspective (operationalized as the latency difference between same and different perspective trials in each environment condition) was smaller in the across-environment than in the within-environment condition. The authors interpreted this finding as evidence for distinct processes governing perspective change within and across environments and proposed an interference account to explain it. Specifically, they argued that when changing perspective within a single environment, conflicts occur between the targets' current and previous relative locations. Such conflicts do not exist when changing perspective across environments (because the targets are different in the current and previous trial), making it faster to change perspective across environments.

The results of Brockmole \& Wang (2003) could have important implications for studies of spatial updating. Spatial updating refers to the process of computing the changed egocentric locations of objects that result from observer or object movement. While spatial updating is rather effortless during physical movement, this process is cognitively demanding and is carried out in a backward manner (i.e., after the movement is completed) when the movement is only imagined (e.g., Avraamides, 2003; Rieser, Guth, \& Hill, 1986). The inferior spatial performance associated with responding from perspectives adopted after imagined movement is typically attributed to the absence of vestibular feedback, proprioceptive information, and/or efferent copy during imagined movements. Recently, an alternative account has been proposed to explain why spatial performance is inferior during imagined compared to physical movement. Specifically, May (1996, 2004; see also Avraamides, Klatzky, Loomis, \& Golledge, 2004, and Wraga, 2003) has suggested that the difficulty (or at least part of the difficulty) when responding from imagined perspectives might be due to conflicts between the physical and imagined egocentric locations of objects. When an observer attempts to localize a target from an imagined position that differs from his/her actual physical position, s/he must only consider object locations relative to his/her imagined position and disregard their locations relative to his/her physical position. As the results of May (2004) and Avraamides et al. (2004) show, suppressing the physical egocentric locations of targets is not always an easy task.

The findings of Brockmole and Wang's (2003) suggest another explanation for the inferior performance evidenced during imagined movements. Specifically, their interference account posits that the problems with reasoning from imagined perspectives might be due (completely or partially) to interference from the objects' previous 
imagined locations. Indeed, if a spatial updating experiment requires that the participant adopt a different imagined perspective in every trial then an imagined targetobject will have a different relative location on every trial. If the imagined location of a target-object is subjected to interference from its imagined location in the previous trial or trials, then performance in the experiment overall will be hindered. This interference account is similar to that proposed by May $(1996 ; 2004)$ but differs from it in that the proposed conflicts are between the previous and current imagined locations of objects and not between the objects' current physical and imagined locations.

While this interference account is plausible, it is based solely on the study by Brockmole and Wang (2003), which had one important design limitation: because each environment in the across-environment block was fixed to a specific yet unique perspective, a sequence of same perspective trials could only occur if the environment also remained unchanged. In essence, there were no true same-perspective/switchenvironment trials.

Furthermore, one finding from that experiment is particularly noteworthy: Latencies were substantially smaller in the across-environment than the within-environment block for both perspective conditions. The interference account cannot explain why maintaining the same perspective was faster in the across condition than the within condition. Maintaining the same perspective in the same environment should not produce any conflicts and it is also free of the temporal cost of environment switching. Intuitively, one should predict this to be the fastest condition.

The present study reexamined the relation between changing perspective and switching environment using a design that allows for the independent manipulation of the two variables. Participants first learned two environments and then performed target-localization trials which probed targets, in a random fashion, from the two environments and four different perspectives. Because spatial updating studies are typically carried out in novel settings (e.g., Farell \& Robertson, 1998; Mou, McNamara, Valiquette, \& Rump, in press; Loomis, Lippa, Klatzky, \& Golledge, 2002), and because our interest is to evaluate the interference hypothesis in the context of spatial updating, the present study uses novel scenes rather than highly familiar ones.

\section{Method}

\subsection{Participants}

A total of 20 students participated in the experiment. Nineteen ( 8 male) participants were students of introductory psychology classes at the University of California, Santa Barbara who participated in exchange for course credit. One female participant was a graduate student in psychology who volunteered to participate. 


\subsection{Stimuli and Apparatus}

The experiment was divided into two phases: a learning phase during which participants studied two virtual rooms, and a testing phase in which their spatial performance was tested.

\subsubsection{Learning Phase}

Participants experienced the virtual rooms via a Virtual Research V8 head-mounted display (HMD; a stereoscopic display with dual $680 \times 480$ resolution LCD panels that refresh at $60 \mathrm{~Hz}$ ). The projectively correct stereo display was rendered by a $2.2 \mathrm{GHz}$ Pentium 4 processor computer with a GeForce 4 graphics card using the Vizard (www.worldviz.com) software package. The simulated viewpoint was continually updated by the participant's head movements. The orientation of the participant's head was tracked by a three axis orientation sensing system (Intersense IS300), and the location of the participant's head was tracked three-dimensionally by a passive optical position sensing system (developed in-house and capable of measuring position with a resolution of 1 part in 30,000, or approximately $0.2 \mathrm{~mm}$ in a $5 \mathrm{~m}$ square workspace). The system latency, or the amount of delay between a participant's head or body motion and the concomitant visual update in the HMD, was $42 \mathrm{~ms}$ maximum.

Two virtual rooms, each with unique wall textures and objects, were used in the learning phase of the experiment (Figure 1). Each virtual room contained four objects that were placed on $1.2 \mathrm{~m}$ tall pillars. The four objects were spaced evenly around the subject (every $90^{\circ}$ ).

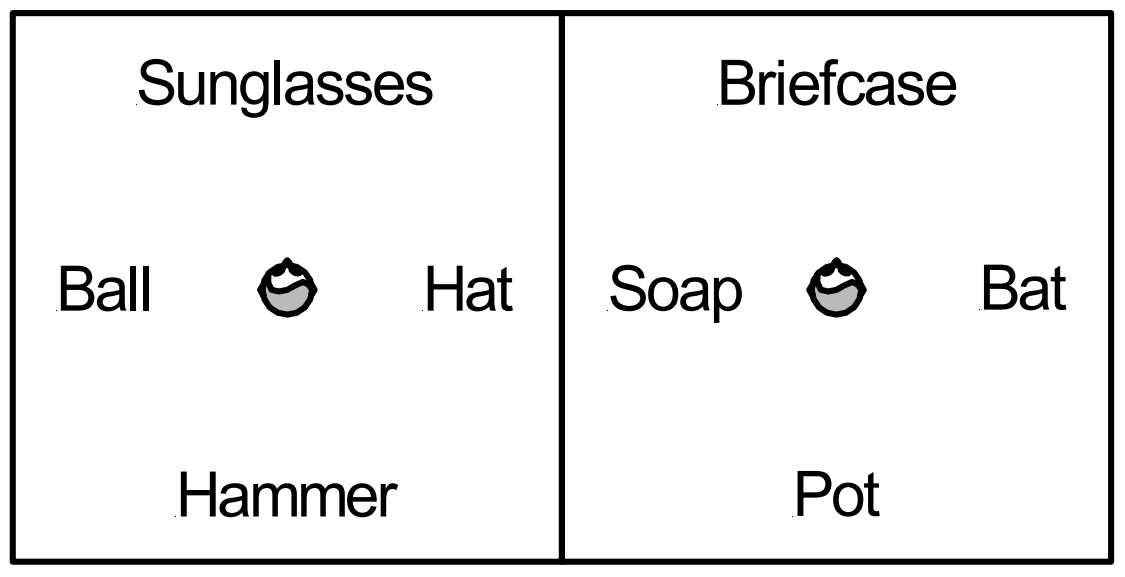

Fig. 1. Schematic diagrams of the layouts of objects in the two virtual rooms

The names of the objects appeared printed on the front of the pillars. One of the rooms appeared to be made of bricks (Figure 2) and the other appeared to be made of wood (Figure 3). This was done to help subjects form distinct representations of the two rooms. 


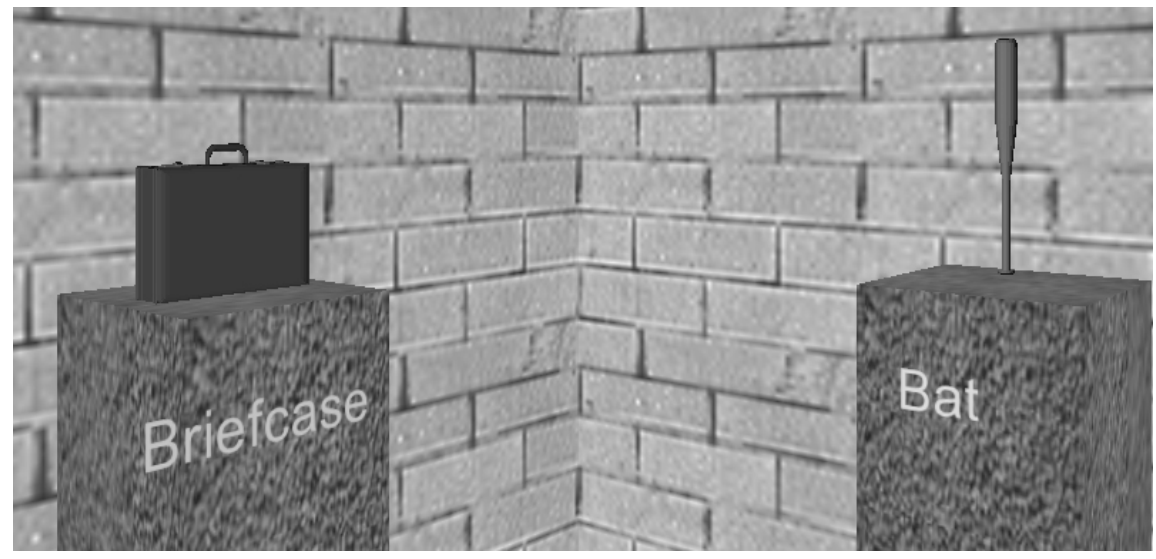

Fig. 2. A view of the brick-walled room

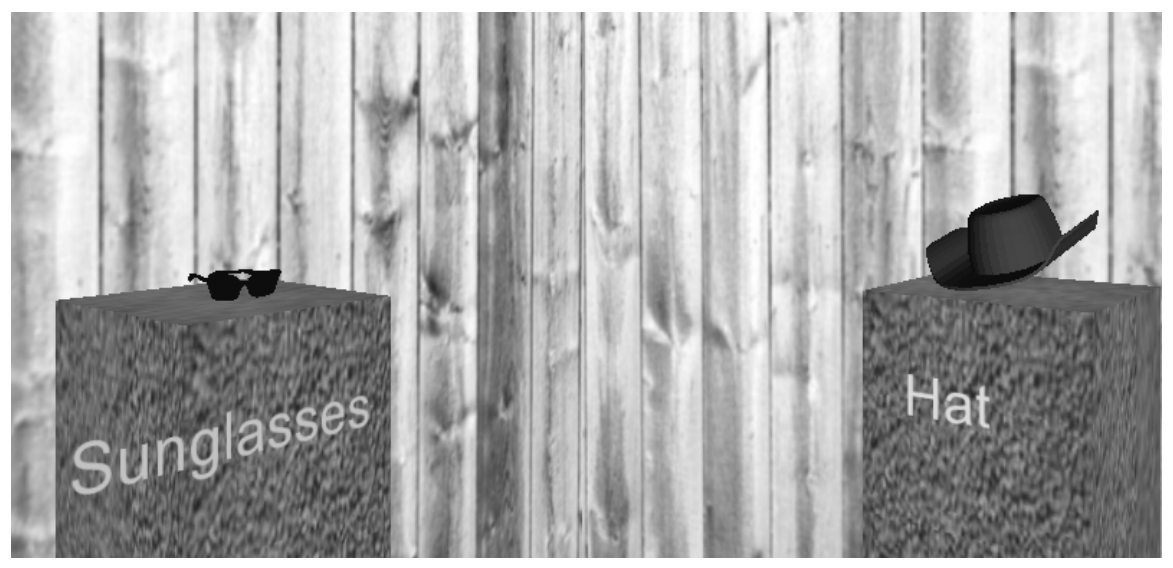

Fig. 3. A view of the wood-walled room

\subsubsection{Testing Phase}

The testing phase was carried out on a laptop computer, also running the Vizard software package. Participants were presented with statements of the form: "Face: $x$, Find $y$ ", where $\mathrm{x}$ and $\mathrm{y}$ were objects from the same room, and were asked to imagine facing object $\mathrm{x}$ and report the location of object $\mathrm{y}$ relative to their facing direction. Responses were collected using four keys on the computer labeled with " $F$ ", "B", "L", and " $\mathrm{R}$ ", corresponding to front, back, left, and right. Timing began with the presentation of the statement and ended when participant pressed a key to indicate an answer. 
The probed object was actually never directly in front of the imagined perspective, so the " $F$ " response was always incorrect. Therefore, there were three object locations that could be probed from each imagined perspective in each environment. Each participant completed 72 trials total, comprised of 3 repetitions of the 12 possible objectperspective combinations, for the 2 different environments. Trials were presented in a different random order for each participant thus producing trials in which the probed room and/or the facing direction were the same or different from the immediately previous trial ${ }^{1}$. Data from the first trial were considered practice and were discarded.

\subsection{Design}

The experiment was a 2 (environment: switch, no switch) $\times 2$ (perspective: same, different) within-subjects factorial design.

\subsection{Procedure}

Participants stood at a fixed location and orientation in the laboratory and wore the HMD. They were placed into the first of the two virtual rooms (the order of room presentation was counterbalanced across participants) and were given unlimited time to study and memorize the four objects that were visible around them. They were instructed to rotate in place in order to examine the room but not to move to a new position in it. Once participants indicated that they had learned the layout, the experimenter tested their memory. To do so, all objects except one were removed from the room and participants were asked to face the only visible object and then point to each of the remaining three objects as probed by the experimenter. This was repeated until participants pointed correctly to all objects from all possible perspectives. Then, participants were asked to adopt their initial facing direction and were placed into the second room and the same procedure was repeated. Upon completion, they were given the option to revisit the first room and refresh their memory for it.

Once participants reported that they knew both virtual rooms very well, they were led to a different laboratory for the testing phase. They were seated in front of a computer at a random physical facing orientation. This phase of the experiment was conducted on a laptop computer, and it was therefore quite easy to manipulate their physical facing direction. After reading the instructions for the testing phase, they began the 72 test trials. As soon as they completed each trial the next one followed with no delay; that is, as soon as they had pressed a key to respond to a statement, the statement for the next trial appeared. Participants were asked to perform the task as fast as they could but without sacrificing accuracy for speed.

\footnotetext{
${ }^{1}$ Because participants experienced the two rooms from the same standpoint and initial facing orientation, we assume that they have noted the correspondence between the two rooms (e.g., left in one room corresponded to left in the other).
} 


\section{Results}

Data from one participant were eliminated from all analyses because of low accuracy $(<40 \%)$. Because accuracy was extremely high in all conditions $(92 \%)$, latency data for correct trials are the primary focus.

Data from trials in which response latency deviated 3 or more standard deviations from each subject's cell mean were considered outliers and were therefore discarded from all analyses. This resulted in eliminating $1.5 \%$ of correct-response trials.

A preliminary analysis of latencies revealed that memories for the two environments were viewpoint-dependent. Participants were $598 \mathrm{~ms}$ faster when judging statements that probed targets from the first view they received upon entering each of the rooms (hereafter referred to as initial viewpoint ${ }^{2}$ ) in the learning phase than from the remaining three perspectives ${ }^{3}$ (hereafter referred to as novel viewpoint), $\underline{\mathrm{t}}(18)=4.16, \mathrm{p}<.001$. More importantly, the pattern of latencies for switching environment and perspective were different for the initial and novel viewpoints. Therefore, separate repeated-measures analyses of variance (ANOVAs) were performed for each viewpoint condition using environment and perspective as factors.

\subsection{Initial viewpoint.}

First, an ANOVA was carried out using the data from trials in which participants imagined adopting the viewpoint that coincided with the first view they got upon entering each of the virtual rooms (the initial viewpoint). This analysis revealed that participants performed the task faster when they maintained the imagined perspective of the previous trial (i.e., when the current and previous trials both tested the initial viewpoint) than when they shifted to a new perspective (i.e., when the current trial tested the initial viewpoint and the previous trial tested a novel viewpoint), $\underline{\mathrm{F}}(1,15)=7.36, \underline{\mathrm{MSE}}=13.98, \underline{\mathrm{p}}<.05$. They were also faster when they remained in the same environment than when they switched, but the difference was not statistically significant.

As seen in Figure 4, changing perspective took longer than switching environment. Furthermore, when participants had to change both environment and perspective, latencies were slightly longer than simply changing perspective, the longer of the two processes.

\footnotetext{
${ }^{2}$ We use the term viewpoint whenever we refer to the distinction between the first view of the room during the learning phase vs. the remaining views. The term perspective is used instead when distinguishing trials based on whether they probed the same or different view (compared to the previous trial) during the test phase.

${ }^{3}$ Latencies for the remaining three perspectives did not differ significantly from each other and were therefore averaged to form a single novel viewpoint mean.
} 


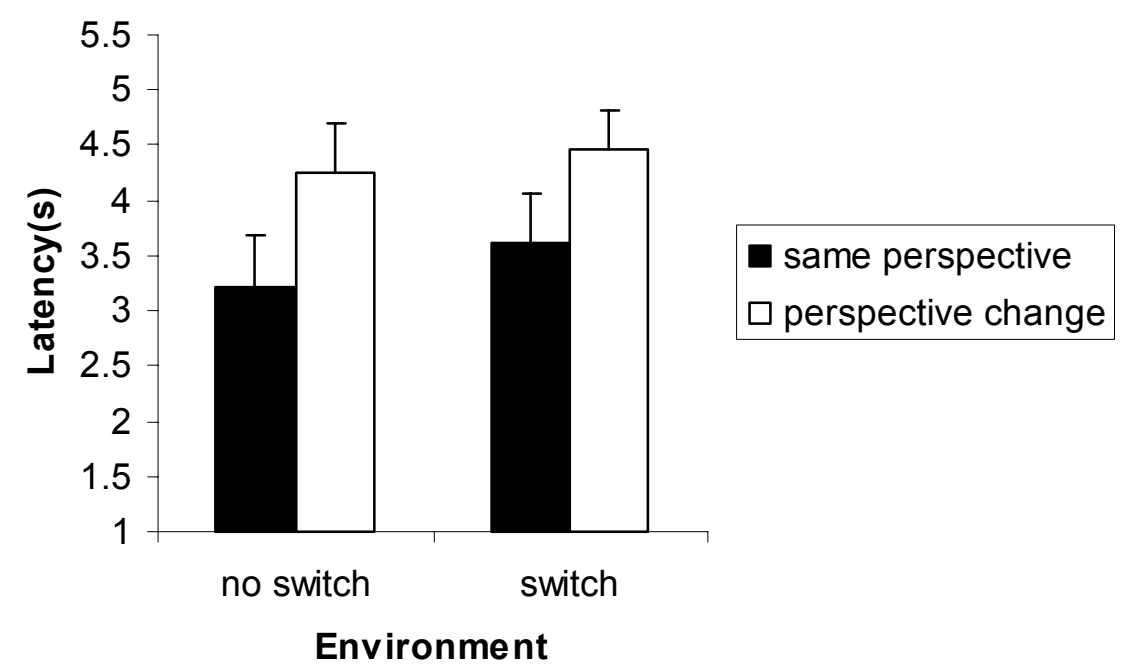

Fig. 4. Initial Viewpoint. Mean latency by perspective and environment condition.Error bars indicate standard errors

\subsection{Novel viewpoint.}

An ANOVA was also carried out for the trials in which participants imagined adopting a view that differed from their first view of the rooms. Results showed that participants were overall faster when they remained in the same environment than when they switched to the other, $\underline{F}(1,18)=27.67, \underline{M S E}=14.47, \underline{p}<.001$. As in the initial viewpoint case, they were faster when they maintained the perspective of the previous trial than when they adopted a different one, $\underline{F}(1,18)=13.13, \underline{M S E}=6.58, \underline{p}<.01$. However, this effect was only present for the same-environment condition. This was supported by a significant environment $\times$ perspective interaction, $\underline{F}(1,18)=3.26$, $\underline{\mathrm{MSE}}=6.21, \underline{\mathrm{p}}<.05$. In contrast to the results from the initial viewpoint analyses, participants were faster changing perspective within than across environments, $\underline{t}(18)=-$ $2.59, \underline{p}<.05$. As seen in Figure 5, switching environment was the longest process, and changing both environment and perspective took slightly longer than only switching environment. 


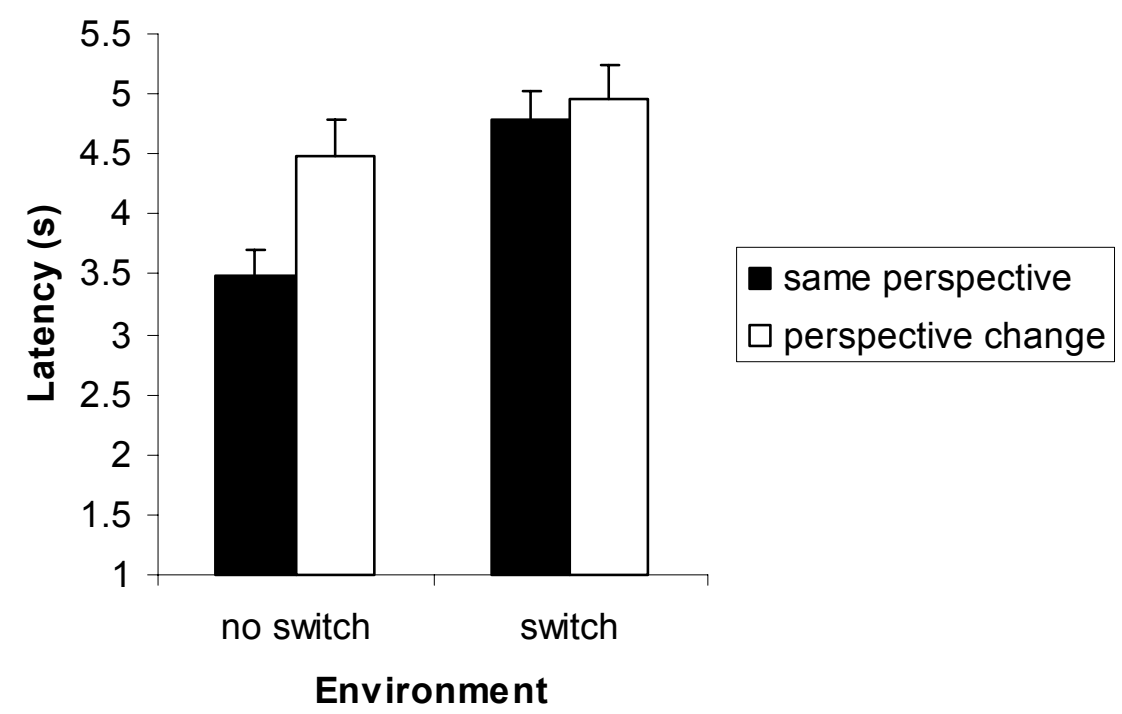

Fig. 5. Novel Viewpoint. Mean latency by perspective and environment condition. Error bars indicate standard errors

\section{Discussion}

The present experiment examined the relation between mentally switching between imagined environments and changing perspective within imagined environments. The results are most compatible with the race model (a parallel-processing model), which predicts that when one has to both switch environment and change perspective, the total time needed is slightly longer than the duration of the longer of the two. When participants mentally adopted the viewpoint with which their spatial memory was aligned to (i.e., the initial viewpoint), changing perspective was the longest process. In contrast, when they adopted a novel viewpoint, switching environment was longer. In both cases there is clear evidence that the costs associated with perspective changing and environment switching are not additive. Instead, results suggest that changing perspective and switching environment are processes that can take place in parallel.

A striking result in the present experiment was that the latency for maintaining the same perspective across environments was substantially longer when people adopted a novel viewpoint than the original. We believe that this was the case because participants represented the scenes in memory from the initial viewpoint and always referred to these representations whenever they had to either change perspective or switch environment. If that was the case, one should also expect the following:

1. Maintaining the same perspective and changing to a new one across environments should not differ when the novel viewpoint is tested because both conditions 
would entail consulting the initial viewpoint. Our results clearly support this hypothesis (see Figure 3).

2. Changing perspective should be faster when the previous trial tested the initial viewpoint. That is, there should be an advantage not only when people change perspective to adopt the initial viewpoint, but also when they change perspective away from it. This, however, should be the case only when the two trials probe the same environment. The results from the initial-viewpoint case (Figure 2) are compatible with this hypothesis: same-perspective trials (that is, trials in which both the current and previous viewpoint were the initial) were faster than change-perspective trials (that is, trials in which the current viewpoint was the initial but the previous one was novel).

To test the hypothesis with novel viewpoints we performed an additional analysis. We coded each perspective-change trial based on whether the immediately preceding trial tested a novel or the initial viewpoint. Results revealed that, within the same environment, the mean latencies for both these conditions differed significantly from the same perspective mean ${ }^{4}$. More importantly, latencies were significantly shorter when the previous trial tested the initial than a novel viewpoint ${ }^{5}$. This result was not obtained when there was an environment switch. This additional analysis further supports the hypothesis that participants maintained viewpoint-dependent representations for the two layouts and that these preferred orientations mediated their performance.

It should be pointed out that this was the case despite the fact that during the learning phase participants had experienced the scenes from four different viewpoints and had even performed localization training trials from all four. Despite this extensive experience with novel viewpoints, participants created and maintained mental representations that were aligned with the initial viewpoint. This finding is consistent with McNamara's (2003) theory of spatial memory which posits that people interpret spatial scenes by assigning a reference frame intrinsic to the layout and update their mental representation only if a later view provides a chance for superior encoding (e.g., Mou \& McNamara, 2002; Shelton \& McNamara, 2001). Extending previous work on viewpoint-dependency (e.g., Christou \& Bülthoff, 1999; Richardson, Montello, \& Hegarty, 1999), we show here that even elaborate experience with a scene from novel viewpoints does not override the initial encoding of the scene.

Despite the evidence for viewpoint-dependent encoding of the spatial layouts, our data do not speak to the question of what reference frame participants used to organize their memories. Our results are compatible with a number of different hypotheses that have been proposed by other researchers. One possibility is that participants have used an egocentric reference frame to encode the location of objects in relation to their bodies as in the case of "spatial frameworks" (e.g., Franklin \& Tversky, 1990), An alternative account is that participants have encoded the allocentric relations of objects using a reference frame that was intrinsic to the scene but was oriented with respect to the first viewing experience (McNamara, 2003). A further possibility is that participants had formed allocentric representations to organize their memory when learning the scenes and imposed an egocentric reference frame when retrieving each spatial relation during the testing phase (Easton \& Sholl, 1995).

\footnotetext{
${ }^{4}$ p's $<.01$

${ }^{5} \mathrm{t}(18)=2.06, \mathrm{p}<.05$
} 
In summary, our results replicated those of Brockmole and Wang (2002) showing that when remaining in the same perspective people are faster when they also remain in the same environment. However, our findings differed from those of Brockmole and Wang (2003) which showed faster performance for changing perspective across than within environments.

Many experimental aspects differed between the present study and that of Brockmole \& Wang (2003), all of which could have produced the discrepant results. For example, while Brockmole and Wang (2003) used environments that were highly familiar to their participants, the present study used environments that were learned by participants just prior to the target-localization trials. It could be the case that interference is greater for familiar than novel environments. There is some evidence in the literature that memories for familiar environment are organized in a different manner than those for novel environments (but see McNamara, 2003 for a critique). The classic study by Evans \& Pezdek (1980), for example, has shown that university students had formed viewpoint-invariant representations for their campus, possibly as a result of having experienced their campus many times from multiple perspectives. In contrast a group of students from a different school who learned the campus only via a map exhibited the typical viewpoint-dependent performance found in the present and many other studies using novel environments (e.g., Presson \& Montello, 1994).

Moreover, in Brockmole \& Wang (2003) the two test environments represented two distinct levels of hierarchical encoding. Participants in that study were tested on their memory for objects located in their office and the building in which their office was located. In contrast, the environments in our study were not nested. It is possible that switching across two presumably unrelated environments is not comparable to switching across two environments that are held in memory hierarchically.

Despite these and other methodological differences between the two studies, the interference account should have, in theory, applied in the present experiment. If it did, this would have cast serious doubts on the conclusions of many studies that use target-localization from imagined perspectives in novel environments. Although our results cannot rule out the presence of interference from the previous relative (mental) locations of targets, they can at least suggest that such interference (if any) is not severe.

In summary, our results show that there are costs for both mentally switching across environments in memory and changing perspective within them, and that these costs are not additive when one needs to mentally switch both environment and perspective.

In our view, the cost for environment-switching results from the need to shift the focus of attention, as defined by Cowan's (1999) theory of working memory, from one spatial representation to the other. Due to the nature of our task (i.e., cascaded trials probing unpredictably one or the other environment), we believe that our participants maintained two separate spatial representations active in their working memory. Nevertheless, only one of them could be at the center of the mental focus at a given point in time. Hence, we propose that the cost for switching from one environment to the other represents the time needed to move the attentional focus

Additionally, we posit that the cost for changing perspective results from updating the stored view (i.e., the initial viewpoint) -- which is activated when the environment is brought into the focus of attention -- to the view that is probed by the trial instruc- 
tion. Updating in this case is not effortless as it is with physical movement. Rather, changing imagined perspective demands cognitive resources as it requires an explicit computation of how the relative locations of objects change as a result of adopting the new imagined perspective.

\section{References}

Avraamides, M. N. (2003). Spatial updating of environments described in texts, Cognitive Psychology, 47, 402-431.

Avraamides, M. N., \& Carlson, R. A. (2003). Egocentric organization of spatial activities in imagined navigation. Memory \& Cognition, 31, 252-261.

Avraamides, M. N., Klatzky, R. L., Loomis, J. M., \& Golledge, R. G. (2004). Use of cognitive vs. perceptual heading during imagined locomotion depends on the response mode. Psychological Science, 15, 403-408

Brockmole, J. R., \& Wang, R. F. (2003). Changing perspective within and across environments. Cognition, 87, B59-B67.

Brockmole, J. R., \& Wang, R. F. (2002). Switching between environmental representations in memory. Cognition, 83, 295-316.

Christou, C.G., and Bülthoff, H.H. (1999). View dependence in scene recognition after active learning. Memory and Cognition, 27, 996-1007.

Cowan, N.(1999). An embedded-process model of working memory. In A. Miyake, \& P. Shah (Eds.), Models of Working Memory: Mechanisms of Active Maintenance and Executive Control (pp. 62-101). New York: Cambridge University Press.

Easton, R. D., \& Sholl, M. J. (1995). Object-array structure, frames of reference, and retrieval of spatial knowledge. Journal of Experimental Psychology: Learning, Memory, \& Cognition, 21, 483-500.

Evans, G. W., \& Pezdek, K. (1980). Cognitive mapping: Knowledge of real-world distance and location information. Journal of Experimental Psychology: Human Learning and Memory, $6,483-500$

Farell, M. J., \& Robertson, I. H. (1998). Mental rotation and the automatic updating of bodycenter spatial relationships. Journal of Experimental Psychology: Learning, Memory, \& Cognition, 24, 227-233.

Franklin, N., \& Tversky, B. (1990). Searching Imagined Environments. Journal of Experimental Psychology: General, 119, 63-76.

Hirtle, S. C., \& Jonides, J. (1985). Evidence of hierarchies in cognitive maps. Memory \& Cognition, 13, 208-217.

Jeannerod, M. (1995). Mental imagery in the motor context. Neuropsychologia, 33, 1419-1432.

Klatzky, R., L, Loomis, J., M, Beall, A., C, Chance, S., S, \& Golledge, R., G. (1998). Spatial updating of self-position and orientation during real, imagined, and virtual locomotion. Psychological Science, 9, 293-298.

Kosslyn, S. M. (1994). Image and brain: The resolution of the imagery debate. Cambridge, MA: MIT Press.

Logan, G. D. (1988). Toward an instance theory of automatization. Psychological Review, 95, 492-527.

Loomis, J. M., Lippa, Y., Klatzky, R. L., \& Golledge, R. G. (2002). Spatial updating of locations specified by 3-D sound and spatial language. Journal of Experimental Psychology: Learning, Memory, \& Cognition, 28, 335-345. 
May, M. (2004). Imaginal perspective switches in remembered environments: Transformation versus interference accounts. Cognitive Psychology, 48, 163-206.

May, M. (1996). Cognitive and embodied modes of spatial imagery. Psychologische Beiträge, $38,418-434$.

McNamara, T. P. (2003). How are the locations of objects in the environment represented in memory? In C. Freksa, W. Brauer, C. Habel, \& K. Wender (Eds.), Spatial cognition III: Routes and navigation, human memory and learning, spatial representation and spatial reasoning (pp. 174-191). Berlin: Springer-Verlag.

McNamara, T. P. (1986). Mental representations of spatial judgments, Cognitive Psychology, $18,87-121$.

Mou, W., \& McNamara, T. P. (2002). Intrinsic frames of reference in spatial memory. Journal of Experimental Psychology: Learning, Memory, \& Cognition, 28, 162-170.

Mou, W., \& McNamara, T. P., Valiquette, C. M, \& Rump, B. (2003). Allocentric and egocentric updating of spatial memories. Journal of Experimental Psychology: Learning, Memory, and Cognition. 28, 162-170.

Presson, C. C, \& Montello, D. R. (1994). Updating after rotational and translational body movements: Coordinate structure of perspective space. Perception, 23, 1447-1455.

Richardson, A. E., Montello, D. R., \& Hegarty, M. (1999). Spatial knowledge acquisition from maps and from navigation in real and virtual environments. Memory \& Cognition, 27, 741750 .

Rieser, J. J. (1989). Access to knowledge of spatial structure at novel points of observation. Journal of Experimental Psychology: Learning, Memory, \& Cognition, 15, 1157-1165.

Rieser, J. J., Guth, D. A, \& Hill, E. W. (1986). Sensitivity to perspective structure while walking without vision. Perception, 15, 173-188.

Shelton, A. L., \& McNamara, T. P. (2001). Systems of spatial reference in human memory. Cognitive Psychology, 43, 274-310.

Stevens, A., \& Coupe, P. (1978). Distortions in judged spatial relations, Cognitive Psychology, $10,422-437$.

Wexler, M., Kosslyn, S. M., \& Berthoz, A. (1998). Motor processes in mental rotation. Cognition, 68, 77-94.

Wraga, M. (2003). Thinking outside the body: An advantage for spatial updating during imagined versus physical self-rotation. Journal of Experimental Psychology: Learning, Memory, \& Cognition, 29, 993-1005.

\section{Acknowledgments}

We thank Roberta Klatzky, Jack Loomis, and Frances Wang for valuable comments and suggestions during the preparation of this paper. We are grateful to Jack Loomis for letting us use his laboratory. 